\title{
NUEVOS MERCADOS DE DESTINO PARA EL ALGODÓN ORGÁNICO DE COLORES
}

\section{NEW DESTINATION MARKETS FOR ORGANIC COLOR COTTON}

Diego Alejandro Wong Cárcamo; Enrique Samuel Amorós Vidales; Edith Alessandra Collantes García; Anthony Christian Alcántara Arias; Patricia Ysabel Gutiérrez Arias; Paula Fernanda Arévalo Guzmán y Carlos Benjamín Quelopana Díaz

Estudiantes de la Universidad Ricardo Palma

Facultad de Ciencias Económicas y Empresariales

Escuela Profesional de Administración de Negocios Globales

\section{RESUMEN}

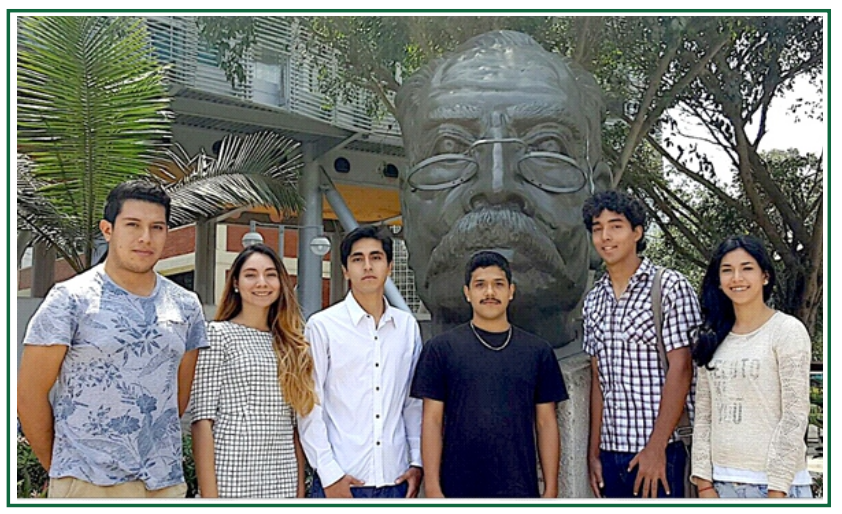

Recepción: 2016-10-05 / Aceptación: 2016-12-16

El objetivo es identificar nuevos mercados de destino para el algodón de colores mediante la investigación de requisitos (criterios) que tienen los posibles nuevos mercados y la capacidad del algodón orgánico de colores para abastecerlos. Se tomaron datos dentro de páginas de inteligencia comercial como SIICEX, cuadros comparativos de investigaciones y tesis. Una vez que se logren resolver los problemas en lo que respecta a cumplir con las metas de calidad del producto según los criterios generales, que son básicamente un nivel aproximado de la calidad de la fibra, cierta resistencia al fuego y diseño de la etiqueta, el producto estaría apto para su exportación, resultando México, Colombia y Chile como posibles nuevos mercados debido a su crecimiento en el sector y los criterios que piden. En conclusión, la exportación del Algodón Nativo presenta grandes oportunidades en el extranjero, gracias a su alto valor agregado y por su potencialidad de industrialización.

Palabras Clave: Exportación, calidad, Algodón Nativo.

\section{ABSTRACT}

The objective is to Identify new destination markets for organic color cotton through the investigation of requirements that possible new markets have and the capacity of the organic color cotton have to provide them. An extraction of data was taken in Business intelligence pages like SIICEX, comparative tables, thesis. Once problems are solved in terms of accomplishing quality product goals according to the general criteria, which are basically an approximate level of fiber quality, some fire resistance and label design, the product would be ready for its exportation. Mexico, Colombia and Chile are coming out as potential new markets due to their growth in the sector and the criteria they request. In sum, the exportation of native cotton shows a great opportunity in the foreign market, thanks to its high valueadded and its potential industrialization.

Keywords: Exportation, quality, native cotton. 


\section{INTRODUCCIÓN}

Fustamante (2012) refiere que el algodón nativo en sus condiciones actuales presenta dificultades para su industrialización e incluso que la baja calidad del mismo no permite la masificación del producto. Sin embargo, el algodón nativo puede lograr un alto valor agregado gracias a la labor artesanal del mismo.

Otro punto clave es la durabilidad del producto, se han encontrado fardos funerarios de la época preincaica, que están intactos a pesar del paso de los años y de las condiciones a las que han sido sometidos, demostrando la excelente calidad que posee el algodón peruano, aun prescindiendo de la industrialización (Millaire y Surette, 2011); esta es una gran fortaleza que tiene especial importancia para los mercados extranjeros.

Debido a la baja industrialización y a la dificultad en el manejo del producto, no se han dado las facilidades para potenciar el producto mismo y lograr así su exportación al extranjero.

El objetivo de este trabajo es identificar nuevos mercados de destino para el algodón de colores, mediante la investigación de los requisitos que tienen los posibles nuevos mercados y la capacidad del algodón orgánico de colores para cumplir con estos requerimientos para abastecerlos.

\section{RESULTADOS}

La división del trabajo se basa en una estructura que define primero los criterios que requiere el algodón orgánico para el ingreso hacia mercados ya conocidos; a continuación, se define la capacidad del producto en relación a su calidad y por último, identifica los criterios básicos que requieren los países que aspiran a ser importadores de algodón, con este propósito, es de suma importancia dividir la investigación de la siguiente manera: Criterios de países importadores, Capacidad y nivel de calidad del algodón y finalmente Criterios de países demandantes.

\section{Criterios de países importadores}

Al analizar los criterios de los principales países que importan productos a base de algodón, se observó la falta de información detallada sobre el algodón nativo en páginas de inteligencia comercial como SIICEX, por consiguiente, no se consideró criterios que exigen las entidades peruanas en esta sección.

Torres (2014) explica que las principales características que deben cumplirse para que el producto ingrese a Estados Unidos de América, son las siguientes:

- Composición de la fibra, se debe indicar el porcentaje de la fibra en masa, en orden descendente según los tipos de fibra que la prenda. Si el porcentaje no llega al $5 \%$, se puede anotar en la etiqueta: Other fibers (Otras fibras). Si la prenda tiene varias secciones de distintas composiciones, cada una se identificará por separado. No es obligatorio señalar en la etiqueta los demás materiales de la prenda (como plástico, metal, otros).

- Datos del responsable del producto (exportador, importador, distribuidor o minorista). En la 
etiqueta deberá constar la razón social de la empresa o el Registered Number (RN), número emitido por la Comisión Federal de Comercio (FTC).

- El país de origen debe aparecer siempre en la cara delantera de la etiqueta, cosida en la parte central del interior del cuello en las prendas que lo posean, o en el caso de prendas sin cuello, en un lugar fácilmente accesible, además, todos estos datos deben estar escritos en idioma inglés.

- Care Labeling Rule. Establece consideraciones para que la etiqueta incluya información acerca del cuidado de las prendas. Cuando no sea posible colocar una etiqueta, sin perjudicar la estética o la calidad, esta podrá constar en una tarjeta colgada de la prenda o en el envoltorio.

Se permite el uso de símbolos aprobados por la ASTM, organismo de normalización de los Estados Unidos de América, cuyo distintivo garantiza el correcto uso de un material o ejecución de un proceso.

- Consumer Product Safety Commission, organizaciones que buscan proteger al consumidor (CPSC), ellos establecen básicamente, que las fibras con las que se confeccionan estos productos no sean altamente inflamables.

- Consumer Product Safety Improvement Act (CPSIA): incluye disposiciones relativas al contenido de plomo, ftalatos y respecto a la seguridad de los juguetes para bebés y niños.

Exige además que los análisis a los productos se realicen en laboratorios acreditados por CPSC y cuenten con una certificación de conformidad. En el caso de Perú, Certintex es el único laboratorio acreditado para hacer estas pruebas a la fecha.

Otro país que del mismo modo, importa algodón peruano a gran escala, es Alemania, quien maneja requerimientos parecidos a los de EE.UU, sin embargo, ellos piden una aprobación de The Textiles and Textile Machinery Standards Committee (TEXTILNORM), una organización encargada de los estándares para el ingreso de sus productos, dichos estándares establecen los requisitos que se requieren por producto textil y uso del mismo, por ejemplo, los requisitos por un producto para bebes.

Mediante estos requisitos básicos se puede estimar los criterios que requerirían los productos a base de algodón nativo para su exportación, sin dejar de lado, que también se debe obtener los certificados que se exigen en nuestro país.

\section{Capacidad y nivel de calidad del algodón}

A diferencia de otras especies de algodón comercial, el algodón nativo presenta colores naturales, esta especie es oriunda de la costa norte del Perú, los tejidos que se producen de esta fibra fueron considerados símbolo de elite por las culturas pre inca.

Vásquez y Pérez (2011) sostienen que los colores del algodón nativo de mayor producción son: Blanco, bombací, colombino, pardo oscuro, crema, pardo claro, fifo o lila, pardo colorado.

Esta es una aproximación a los colores ya que no se tiene una paleta definida y muchas veces 
las prácticas de cosechas inadecuadas hacen que una mayor exposición de las fibras al sol modifique el color original del algodón, volviéndolo más opaco, afectando el hilado porque no hay uniformidad de colores. Existe además el color verde que no es muy común.

El análisis de la fibra se realiza para determinar los parámetros de su calidad, es decir las características físicas, químicas y mecánicas de las fibras textiles que se toman como referencia para determinar la calidad.

\section{Existe un estudio realizado por la Organización} Caritas y la Universidad de Lima que muestra los niveles y parámetros de calidad del algodón nativo de colores, a continuación explicaremos cada parámetro:

- El micronaire es la medida de la finura del algodón que se hace con relación al mayor o menor diámetro.

- La uniformidad de la longitud mide la proporción de fibras de una barba (muestra) con una longitud igual o superior al promedio.

- La longitud de fibra se refiere a la longitud promedio de una porción típica de fibras de una muestra de algodón.

- La resistencia de la fibra se define como la resistencia que oponen al ser sometidas a una tensión.

- La elongación es la medida del comportamiento elástico de las fibras durante la medición de la longitud y resistencia a la tracción.

\section{Tabla 1}

Resultados de la evaluación de la fibra del Algodón Nativo - 2011 - Elaboración realizada por la organización Caritas del Perú y la Universidad de Lima

\begin{tabular}{lccccc} 
COLOR & $\begin{array}{c}\text { Micronaire } \\
\text { (finure) }\end{array}$ & $\begin{array}{c}\text { Longitud } \\
(\mathbf{m m})\end{array}$ & $\begin{array}{c}\text { Indice de } \\
\text { Uniformidad }\end{array}$ & $\begin{array}{c}\text { Resistencia } \\
(\mathbf{N})\end{array}$ & Elongación \\
\hline Crema & 6,58 & 23,32 & 81,3 & 32,1 & 11,7 \\
\hline Pardo & 5,96 & 24,66 & 77,4 & 25,6 & 12,0 \\
\hline Verde & 2,89 & 22,22 & 74,5 & 23,9 & 9,4 \\
\hline Marrón & 4,22 & 23,55 & 77,7 & 25,6 & 15,0 \\
\hline Pardo Claro & 5,63 & 20,73 & 70,5 & 21,5 & 18,0 \\
\hline
\end{tabular}

Debemos enfatizar que no todos los colores de esta especie de algodón están aptos para ser utilizados en procesos productivos que demanden el uso de tecnología industrializada, por esta razón, de los colores evaluados, únicamente los colores pardo y crema se encuentran idóneos para hacer hilos de gran calidad y finura, ya que la resistencia, longitud y elongación son las más altas a comparación de los otros colores.

Sin embargo, se realizaron pruebas que permitieron crear un hibrido que tendría los estándares necesarios para que los demás colores del algodón nativo, puedan alcanzar un nivel de calidad adecuado para una producción industrializada del mismo. Dicha mezcla comprende al ya mencionado algodón nativo y al algodón Tangüis. 
Wong, D.A., Amorós, E.S., Collantes, E.A., Alcántara, A.C., Gutiérrez, P.Y., Arevalo, P.F. y Quelopana, C.B.

\section{Tabla 2}

Características de hilos artesanales obtenidos a partir de Algodón nativo y algodón Tangüis mezclados en diferentes proporciones - 2012 - Elaboración realizada por docentes de la carrera de ingeniera industrial de la Universidad de Lima

\begin{tabular}{|c|c|c|c|c|c|c|}
\hline ANÁLISIS & $\begin{array}{c}\text { Porcentaje } \\
\text { de } \\
\text { Mezcla }\end{array}$ & $\begin{array}{c}75 \% \text { Algodón } \\
\text { Nativo / 25\% } \\
\text { Tangüis }\end{array}$ & $\begin{array}{c}\text { Porcentaje } \\
\text { de } \\
\text { Mezcla }\end{array}$ & $\begin{array}{c}50 \% \text { Algodón } \\
\text { Nativo / } 50 \% \\
\text { Tangüis }\end{array}$ & $\begin{array}{c}\text { Porcentaje } \\
\text { de } \\
\text { Mezcla }\end{array}$ & $\begin{array}{c}25 \% \text { Algodón } \\
\text { Nativo / } 75 \% \\
\text { Tangüis }\end{array}$ \\
\hline & $\begin{array}{c}\text { Hilatura } \\
\text { normal }\end{array}$ & $\begin{array}{l}\text { Hilatura } \\
\text { fina }\end{array}$ & $\begin{array}{l}\text { Hilatura } \\
\text { fina }\end{array}$ & $\begin{array}{c}\text { Hilatura } \\
\text { normal }\end{array}$ & $\begin{array}{l}\text { Hilatura } \\
\text { fina }\end{array}$ & $\begin{array}{c}\text { Hilatura } \\
\text { normal }\end{array}$ \\
\hline Recistencia (N) & 615,4 & 838,2 & 838,2 & 582,6 & ---- & 583,1 \\
\hline Elongación (\%) & 11,6 & 8,2 & 8,2 & 8,2 & 3,0 & 10,1 \\
\hline Titulo (Ne) & 4,25 & 4,07 & 4,07 & 4,07 & 3,48 & 4,25 \\
\hline
\end{tabular}

Este estudio determinó que se mejoran las características físicas del hilo de algodón nativo de color al procesarlo mezclándolo con algodón Tangüis, encontrándose por ajuste de curva un porcentaje óptimo de mezcla en un rango de $60 \%$ y $40 \%$ de ANC y Tangüis (Cortijo de Arbildo y Cancio, 2012)

\section{Criterios de países demandantes}

Considerando que no existe información sobre países que requieren el algodón nativo de colores, se tomaron en cuenta criterios generales para el ingreso de productos textiles como los requerimientos de etiqueta, el seguimiento de tendencias, características culturales. No se tomaron en cuenta los requisitos de calidad por ser muy parecidos a los de los países importadores.

- Para ingresar al mercado internacional se deben elaborar diseños de vanguardia en distintos colores, aprovechando que el color de las prendas de algodón de colores no se desvanece, incluso se vuelven más intensas después del lavado, se debería considerar diseños que sigan las tendencias actuales dependiendo de los países a los que se dirija.

- Los requisitos de las etiquetas de las prendas son básicamente los mismos por cada país, estos son: composición de la fibra, país de origen, tipo de lavado, marca, nombre del producto, información de la empresa o persona jurídica. La mayoría de los datos que figuran en la etiqueta, no cambian de país en país, solo hay que a hacer la traducción de estos al idioma que corresponda.

- Para las ventas de los productos en el extranjero, Las características culturales de cada país son influyentes por lo tanto, se deben tener en cuenta los principales rasgos culturales, para tomar decisiones acertadas: la religión del país (para la selección de los colores), subculturas influyentes (para distinguir y segmentar el mercado), así como las tendencias de cada país (para la selección de diseños) 


\section{DISCUSIÓN}

El algodón nativo tiene grandes oportunidades en el mercado extranjero, teniendo en cuenta que si la producción de este tipo de algodón llega a los estándares requeri-dos para ingresar al mercado estadounidense, este alcanzaría un gran logro, pues los requisitos básicos para este mercado, se piden también para ingresar a la mayoría de países, ya que dichos estándares solo varían en relación a ciertas instituciones públicas de cada país.

Debido a la baja industrialización del producto, sería recomendable un control de calidad interno que siga las pautas establecidas por las organizaciones extranjeras, así como los requisitos básicos que pidan los nuevos mercados de destino.

En relación a la cantidad y calidad de la oferta, podemos ver que se abre una gran puerta al mercado internacional, a través del uso de híbridos que contengan mezclas del algodón nativo y otro, encontramos en nuestra investigación que el Tangüis, representa una excelente opción, si se usan porcentajes mayores de algodón nativo y un menor porcentaje del otro algodón, con lo que aumenta considerablemente la resistencia y fineza de la fibra, a comparación de los parámetros del algodón nativo solo, sin embargo, esta mezcla puede presentar problemas para su exposición y venta en el mercado extranjero, ya que pierde valor agregado por la mezcla propia, además en el proceso de etiquetado se tendría que colocar los porcentajes del algodón, lo puede generar desconfianza por parte del consumidor al no conocer el producto, incluso pueden generar problemas para realizar bien los cálculos de los porcentajes de cada tipo de algodón, debido a la baja industrialización del producto, sin embargo esta baja industrialización permite un uso de programas como el Exporta Fácil para el comercio del producto, porque cumpliría los requisitos del método.

Para contrarrestar las posibles desconfianzas del consumidor, los empresarios algodoneros peruanos participan en las ferias internacionales como la ExpoComer de Panamá, que reúne tanto a inversionistas como emprendedores de diversos rubros empresariales, a través de estas ferias se presentan las fortalezas y diferencias que hacen del algodón nativo un nuevo producto de alta calidad.

Por otro lado, encontramos que no existe una partida arancelaria para el algodón nativo, lo que representa una traba burocrática para los empresarios algodoneros peruanos.

Una vez que se logren resolver estos problemas y logremos alcanzar los estándares internacionales de calidad que se requieren para el algodón nativo de colores, según los criterios generales expuestos en este documento, el producto estaría apto para su exportación y se podrían aprovechar tanto nuevos mercados en donde no se exportan textiles, como países donde la demanda de textiles es alta, entre ellos México, Colombia y Chile cuya demanda destaca en relación a nuestras propias exportaciones de algodón normal, y los requisitos que piden son exactamente los generales que ya hemos alcanzado, con ciertas variaciones que se relacionan a normas de etiquetado, mas no en la calidad del producto. 


\section{REFERENCIAS}

Cortijo de Arbildo, D. \& Cancio, R. (2012). Innovación tecnológica para recuperar el algodon nativo de color. Revista de la Universidad de Lima, 30, pp. 225-245. Recuperado el 2016 -11- 15, desde http://www.ulima. edu.pe/sites/default/files/research/files/innovacion_ tecnologica_para_recuperar_el_algodon_nativo_de_ color_revista_ingenieria_industrial.pdf

Fustamante, K. (2012). Procesamiento artesanal del Algodón Nativo: Una actividad economica viable o solo una tradición. (Tesis para optar el grado de Magister en Biocomercio y Desarrollo Sostenible). Pontificia Universidad Católica del Perú, Lima, Perú.

Honores, A. \& Rodriguez, E. (2007). Etnobotánica del Perú Pre - Hispánico. Trujillo: Ediciones Herbarium Truxillense.

Millaire, J. \& Surette, F. (2011). Un fardo funerario procedente de Huaca Santa Clara, Valle de Virú (ca. 1150 a. D.). Bulletin de I'Institut français d'études andines, 40(2), pp.289-305.Recuperado el 2016/11/15, desde http://www.redalyc.org/pdf/126/12622727003.pdf

Torres, L. (2014). Marcas peruanas que visten al mundo. Exportando.pe. Lima: Siicex.

Vásquez, P. \& Pérez, P. (2011). Revalorando un cultivo ancestral: Algodón Nativo fibra de calidad para la industria. Lima: Caritas del Perú.

\section{Correo electrónico para correspondencia}

diegowongcarcamo@hotmail.com 